Bull. Austral. Math. Soc.

$53 \mathrm{C} 42,53 \mathrm{C} 20$

VOL. $70(2004) \quad[35-44]$

\title{
THE CURVATURE AND TOPOLOGICAL PROPERTIES OF HYPERSURFACES WITH CONSTANT SCALAR CURVATURE
}

\author{
Shu Shichang and LiU Sanyang
}

In this paper, we consider $n(n \geqslant 3)$-dimensional compact oriented connected hypersurfaces with constant scalar curvature $n(n-1) r$ in the unit sphere $S^{n+1}(1)$. We prove that, if $r \geqslant(n-2) /(n-1)$ and $S \leqslant(n-1)(n(r-1)+2) /(n-2)$ $+(n-2) /(n(r-1)+2)$, then either $M$ is diffeomorphic to a spherical space form if $n=3$; or $M$ is homeomorphic to a sphere if $n \geqslant 4$; or $M$ is isometric to the Riemannian product $S^{1}\left(\sqrt{1-c^{2}}\right) \times S^{n-1}(c)$, where $c^{2}=(n-2) /(n r)$ and $S$ is the squared norm of the second fundamental form of $M$.

\section{INTRODUCTION}

Let $M$ be an $n$-dimensional hypersurface in the unit sphere $S^{n+1}(1)$ of dimension $n+1$. Suppose the scalar curvature $n(n-1) r$ of $M$ is constant and $r \geqslant 1$. Cheng and Yau [3] and $\mathrm{Li}$ [7] obtained some characterisation theorems in terms of the sectional curvature or the squared norm of the second fundamental form of $M$ respectively. We should notice that the condition $r \geqslant 1$ plays an essential role in the proofs of their theorems. On the other hand, for any $0<c<1$, by considering the standard immersions $S^{n-1}(c)$ $\subset R^{n}, S^{1}\left(\sqrt{1-c^{2}}\right) \subset R^{2}$ and taking the Riemannian product immersion $S^{1}\left(\sqrt{1-c^{2}}\right)$ $\times S^{n-1}(c) \hookrightarrow R^{2} \times R^{n}$, we obtain a hypersurface $S^{1}\left(\sqrt{1-c^{2}}\right) \times S^{n-1}(c)$ in $S^{n+1}(1)$ with constant scalar curvature $n(n-1) r$, where $r=(n-2) /\left(n c^{2}\right)>1-(2 / n)$. Hence, not all Riemannian products $S^{1}\left(\sqrt{1-c^{2}}\right) \times S^{n-1}(c)$ are covered by the results of $[3,7]$; since the Riemannian product $S^{1}\left(\sqrt{1-c^{2}}\right) \times S^{n-1}(c)$ has only two distinct principal curvatures and its scalar curvature $n(n-1) r$ is constant and satisfies $r>1-(2 / n)$. Hence, Cheng [4] asked the following interesting problem:

Problem 1. ([4]). Let $M$ be an $n$-dimensional compact hypersurface with constant scalar curvature $n(n-1) r$ in $S^{n+1}(1)$. If $r>1-(2 / n)$ and $S \leqslant(n-1)$ $(n(r-1)+2) /(n-2)+(n-2) /(n(r-1)+2)$, then is $M$ isometric to either a totally umbilical hypersurface or the Riemannian product $S^{1}\left(\sqrt{1-c^{2}}\right) \times S^{n-1}(c)$ ?

Cheng [4] said that when $r=(n-2) /(n-1)$, he answered the Problem 1 affirmatively. For the general case, Problem 1 is still open.

\footnotetext{
Received 30th September, 2003

This work is supported in part by the Natural Science Foundation of China.
}

Copyright Clearance Centre, Inc. Serial-fee code: 0004-9727/04 \$A2.00+0.00. 
In this paper, we try to solve Problem 1. We shall give a topological answer, which relies on the Lawson-Simons formula $([8])$ for the nonexistence of stable $k$-currents, which enables us to eliminate the homology groups and to show $M$ is a homology sphere. We prove the following

THEOREM. Let $M$ be an $n(n \geqslant 3)$-dimensional compact oriented connected hypersurface with constant scalar curvature $n(n-1) r$ in $S^{n+1}(1)$. If $r \geqslant(n-2) /(n-1)$ and $S \leqslant(n-1)(n(r-1)+2) /(n-2)+(n-2) /(n(r-1)+2)$, then either $M$ is diffeomorphic to a spherical space form if $n=3$; or $M$ is homeomorphic to a sphere if $n \geqslant 4$; or $M$ is isometric to the Riemannian product $S^{1}\left(\sqrt{1-c^{2}}\right) \times S^{n-1}(c)$, where $c^{2}=(n-2) /(n r)$.

\section{Preliminaries}

Let $M$, be an $n$-dimensional hypersurface in a unit sphere $S^{n+1}(1)$ with constant scalar curvature $n(n-1) r$. We take a local orthonormal frame field $e_{1}, \cdots, e_{n+1}$ in $S^{n+1}(1)$, restricted to $M, e_{1}, \cdots, e_{n}$ are tangent to $M$. Let $\omega_{1}, \cdots, \omega_{n+1}$ be the dual coframe fields in $S^{n+1}(1)$. We use the following convention on the ranges of indices: $1 \leqslant A, B, C, \cdots, \leqslant n+1 ; 1 \leqslant i, j, k, \cdots, \leqslant n$. The struture equations of $S^{n+1}(1)$ are given by

$$
\begin{aligned}
d \omega_{A} & =-\sum_{B=1}^{n+1} \omega_{A B} \wedge \omega_{B}, \quad \omega_{A B}+\omega_{B A}=0, \\
d \omega_{A B} & =-\sum_{C=1}^{n+1} \omega_{A C} \wedge \omega_{C B}+\frac{1}{2} \sum_{C, D=1}^{n+1} R_{A B C D} \omega_{C} \wedge \omega_{D}, \\
R_{A B C D} & =\left(\delta_{A C} \delta_{B D}-\delta_{A D} \delta_{B C}\right),
\end{aligned}
$$

where $R_{A B C D}$ denotes the components of the curvature tensor of $S^{n+1}(1)$. Then, in $M$

$$
\omega_{n+1}=0
$$

It follows from Cartan's Lemma that

$$
\omega_{n+1 i}=\sum_{j} h_{i j} \omega_{j}, h_{i j}=h_{j i} \text {. }
$$

The second fundamental form $B$ and the mean curvature of $M$ are defined by $B=\sum_{i, j} h_{i j} \omega_{i} \omega_{j} e_{n+1}$ and $n H=\sum_{i} h_{i i}$, respectively. The structure equations of $M$ are given by

$$
\begin{aligned}
d \omega_{i} & =-\sum_{k=1}^{n} \omega_{i k} \wedge \omega_{k}, \omega_{i j}+\omega_{j i}=0, \\
d \omega_{i j} & =-\sum_{k=1}^{n} \omega_{i k} \wedge \omega_{k j}+\frac{1}{2} \sum_{k, l=1}^{n} R_{i j k l} \omega_{k} \wedge \omega_{l}, \\
R_{i j k l} & =\left(\delta_{i k} \delta_{j l}-\delta_{i l} \delta_{j k}\right)+\left(h_{i k} h_{j l}-h_{i l} h_{j k}\right),
\end{aligned}
$$


where $R_{i j k l}$ denotes the components of the Riemannian curvature tensor of $M$. From the above equation, we have

$$
n(n-1) r=n(n-1)+n^{2} H^{2}-S
$$

where $n(n-1) r$ is the scalar curvature of $M$ and $S=\sum_{i, j=1}^{n} h_{i j}^{2}$ is the squared norm of the second fundamental form of $M$.

The Codazzi equation and the Ricci identities are

$$
\begin{aligned}
h_{i j k} & =h_{i k j}, \\
h_{i j k l}-h_{i j l k} & =\sum_{m} h_{i m} R_{m j k l}+\sum_{m} h_{j m} R_{m i k l},
\end{aligned}
$$

where the first and the second covariant derivatives of $h_{i j}$ are defined by

$$
\begin{aligned}
& \sum_{k} h_{i j k} \omega_{k}=d h_{i j}-\sum_{k} h_{i k} \omega_{k j}-\sum_{k} h_{j k} \omega_{k i}, \\
& \sum_{l} h_{i j k l} \omega_{l}=d h_{i j k}-\sum_{l} h_{i j l} \omega_{l k}-\sum_{l} h_{i l k} \omega_{l j}-\sum_{l} h_{l j k} \omega_{l i} .
\end{aligned}
$$

We need the following Lemmas.

LEMMA 1. ([5] or [9].) Let $A=\left(a_{i j}\right), i, j=1, \cdots, n$ be a symmetric $(n \times n)$ matrix, $n \geqslant 2$. Assume that $A_{1}=\operatorname{tr} A, A_{2}=\sum_{i, j}\left(a_{i j}\right)^{2}$, then

$$
\begin{aligned}
& \sum_{i}\left(a_{i n}\right)^{2}-A_{1} a_{n n} \\
& \quad \leqslant \frac{1}{n^{2}}\left\{n(n-1) A_{2}+(n-2) \sqrt{n-1}\left|A_{1}\right| \sqrt{n A_{2}-\left(A_{1}\right)^{2}}-2(n-1)\left(A_{1}\right)^{2}\right\} .
\end{aligned}
$$

We prove the following algebraic Lemma by a simple and direct method.

LEMmA 2. Let $A=\left(a_{i j}\right), i, j=1, \cdots, n$ be a symmetric $(n \times n)$ matrix, $p+q$ $=n, p, q \geqslant 2$ are positive integers. Assume that $\sum_{s=1}^{p} a_{s s}+\sum_{t=p+1}^{n} a_{t t}=A_{1}, \sum_{i=1}^{n}\left(a_{i i}\right)^{2}=\tilde{A}_{2}$. Then

$$
\begin{aligned}
& \left(\sum_{s=1}^{p} a_{s s}\right)^{2}-A_{1}\left(\sum_{s=1}^{p} a_{s s}\right) \\
& \quad \leqslant \frac{1}{n^{2}}\left\{p q n \widetilde{A}_{2}-2 p q\left(A_{1}\right)^{2}+|p-q| \sqrt{p q}\left|A_{1}\right| \sqrt{n \widetilde{A}_{2}-\left(A_{1}\right)^{2}}\right\} .
\end{aligned}
$$

Proof: By Cauchy-Schwarz inequality we obtain

$$
\begin{aligned}
\tilde{A}_{2} & =\sum_{s=1}^{p}\left(a_{s s}\right)^{2}+\sum_{t=p+1}^{n}\left(a_{t t}\right)^{2} \geqslant \frac{1}{p}\left(\sum_{s=1}^{p} a_{s s}\right)^{2}+\frac{1}{q}\left(\sum_{t=p+1}^{n} a_{t t}\right)^{2} \\
& =\frac{n}{p q}\left(\sum_{s=1}^{p} a_{s s}\right)^{2}-\frac{2}{q} A_{1}\left(\sum_{s=1}^{p} a_{s s}\right)+\frac{1}{q}\left(A_{1}\right)^{2} .
\end{aligned}
$$


Hence

$$
\left(\sum_{s=1}^{p} a_{s s}\right)^{2}-\frac{2 p}{n} A_{1}\left(\sum_{s=1}^{p} a_{s s}\right)+\frac{p}{n}\left(A_{1}\right)^{2}-\frac{p q}{n} \widetilde{A}_{2} \leqslant 0 .
$$

From (2.17) we have

$$
\frac{p A_{1}}{n}-\frac{\sqrt{p q}}{n} \sqrt{n \widetilde{A}_{2}-\left(A_{1}\right)^{2}} \leqslant \sum_{s=1}^{p} a_{s s} \leqslant \frac{p A_{1}}{n}+\frac{\sqrt{p q}}{n} \sqrt{n \widetilde{A}_{2}-\left(A_{1}\right)^{2}} .
$$

From (2.17) we also have

$$
\left(\sum_{s=1}^{p} a_{s s}\right)^{2}-A_{1}\left(\sum_{s=1}^{p} a_{s s}\right) \leqslant \frac{p q}{n} \widetilde{A}_{2}-\frac{p}{n}\left(A_{1}\right)^{2}+\frac{p-q}{n} A_{1}\left(\sum_{s=1}^{p} a_{s s}\right) .
$$

By (2.18) we have

$$
\begin{aligned}
\left(\sum_{s=1}^{p} a_{s s}\right)^{2}-A_{1}\left(\sum_{s=1}^{p} a_{s s}\right) \leqslant \frac{p q}{n} \widetilde{A}_{2} & -\frac{p}{n}\left(A_{1}\right)^{2} \\
& +\frac{(p-q) p}{n^{2}}\left(A_{1}\right)^{2}+\left|\frac{p-q}{n} A_{1}\right| \frac{\sqrt{p q}}{n} \sqrt{n \widetilde{A}_{2}-\left(A_{1}\right)^{2}} .
\end{aligned}
$$

Hence (2.15) holds. Lemma 2 is proved.

From [8] we have the following result.

LEMMA 3. ([8].) Let $M$ be a compact $n$-dimensional submanifold of the unit sphere $S^{n+m}(1)$ with second fundamental form $B$, and let $p, q$ be positive integers such that $1<p, q<n-1, p+q=n$. If the inequality

$$
\sum_{s=1}^{p} \sum_{t=p+1}^{n}\left(2\left|B\left(e_{s}, e_{t}\right)\right|^{2}-\left\langle B\left(e_{s}, e_{s}\right), B\left(e_{t}, e_{t}\right)\right\rangle\right)<p q
$$

holds for any point of $M$ and any local orthonormal frame field $\left\{e_{s}, e_{t}\right\}$ on $M$, then $H_{p}(M, Z)=H_{q}(M, Z)=0$, where $H_{s}(M, Z)$ denotes the $s$-th homology group of $M$ whth integer coefficients.

REMARK. Lemma 3 is ture for general submanifold with any codimension $m$ of $S^{n+m}(c)$, of course is true for hypersurface of $S^{n+1}(1)$.

LEMMA 4. ([11] or [1].) Let $\mu_{i}, i=1, \cdots, n$ be real numbers such that $\sum_{i} \mu_{i}=0$ and $\sum_{i} \mu_{i}^{2}=\beta^{2}, \beta=$ constant $\geqslant 0$, then

$$
-\frac{n-2}{\sqrt{n(n-1)}} \beta^{3} \leqslant \sum_{i} \mu_{i}^{3} \leqslant \frac{n-2}{\sqrt{n(n-1)}} \beta^{3},
$$

and the equality holds in (2.21) if and only if at least $(n-1)$ of the $\mu_{i}$ are equal.

From Aubin [2, see p. 344], we have.

LEMMA 5. ([2].) If the Ricci curvature of a compact Riemannian manifold is nonnegative and positive at somewhere, then the manifold carries a metric with positive Ricci curvature. 


\section{Proof of TheOREM}

Proof: For a given point $P \in M$, we choose an orthonormal frame field $e_{1}, \cdots, e_{n}$, such that $h_{i j}=\lambda_{i} \delta_{i j}$. From (2.10) and (2.11) by a standard calculation we have

$$
\frac{1}{2} \Delta S=\sum_{i, j, k} h_{i j k}^{2}+\sum_{i} \lambda_{i}(n H)_{i i}+\frac{1}{2} \sum_{i, j} R_{i j i j}\left(\lambda_{i}-\lambda_{j}\right)^{2} .
$$

Let $\mu_{i}=\lambda_{i}-H$ and $f^{2}=\sum_{i} \mu_{i}^{2}$, we have

$$
\begin{aligned}
& \sum_{i} \mu_{i}=0, f^{2}=S-n H^{2}, \\
& \sum_{i} \lambda_{i}^{3}=\sum_{i} \mu_{i}^{3}+3 H f^{2}+n H^{3} .
\end{aligned}
$$

From (2.8) we get $R_{i j i j}=1+\lambda_{i} \lambda_{j}$, putting this into (3.1), by (3.2),(3.3) we get

$$
\begin{aligned}
\frac{1}{2} \triangle S & =\sum_{i, j, k} h_{i j k}^{2}+\sum_{i} \lambda_{i}(n H)_{i i}+\frac{1}{2} \sum_{i, j}\left(1+\lambda_{i} \lambda_{j}\right)\left(\lambda_{i}-\lambda_{j}\right)^{2} \\
& =\sum_{i, j, k} h_{i j k}^{2}+\sum_{i} \lambda_{i}(n H)_{i i}+n S-n^{2} H^{2}-S^{2}+n H \sum_{i} \lambda_{i}^{3} \\
& =\sum_{i, j, k} h_{i j k}^{2}+\sum_{i} \lambda_{i}(n H)_{i i}+n f^{2}+n H^{2} f^{2}-f^{4}+n H \sum_{i} \mu_{i}^{3} .
\end{aligned}
$$

By Lemma 4, we get

$$
\frac{1}{2} \triangle S \geqslant \sum_{i, j, k} h_{i j k}^{2}+\sum_{i} \lambda_{i}(n H)_{i i}+f^{2}\left\{n+n H^{2}-f^{2}-n|H| \frac{n-2}{\sqrt{n(n-1)}} f\right\} .
$$

We denote

$$
P_{H}(f)=n+n H^{2}-f^{2}-n|H| \frac{n-2}{\sqrt{n(n-1)}} f .
$$

From (2.9) we know $f^{2}=S-n H^{2}=(n-1) / n[S-n(r-1)]$, then by (2.9) we write $P_{H}(f)$ as

$$
\begin{aligned}
P_{r}(S)=n+n(r-1)-\frac{n-2}{n}[ & S-n(r-1)] \\
& -\frac{n-2}{n} \sqrt{[n(n-1)(r-1)+S][S-n(r-1)]}
\end{aligned}
$$

Hence(3.5) can be written as

$$
\frac{1}{2} \triangle S \geqslant \sum_{i, j, k} h_{i j k}^{2}+\sum_{i} \lambda_{i}(n H)_{i i}+\frac{n-1}{n}[S-n(r-1)] P_{r}(S)
$$


On the other hand, for any point and any unit vector $v \in T_{P} M$, we choose a local orthonormal frame field $e_{1}, \cdots, e_{n}$ such that $e_{n}=v$, we have from Gauss equation (2.8) that the Ricci curvature $\operatorname{Ric}(v, v)$ of $M$ with respect to $v$ is expressed as

$$
\operatorname{Ric}(v, v)=(n-1)+n H h_{n n}-\sum_{i=1}^{n} h_{i n}^{2} .
$$

By Lemma 1,(3.6) and (3.7) we get

$$
\operatorname{Ric}(v, v) \geqslant \frac{n-1}{n}\left[n+n H^{2}-\frac{n(n-2)}{\sqrt{n(n-1)}}|H| f-f^{2}\right]=\frac{n-1}{n} P_{r}(S)
$$

When $S \leqslant(n-1)(n(r-1)+2) /(n-2)+(n-2) /(n(r-1)+2)$, we know this is equivalent to

$$
\left\{n+n(r-1)-\frac{n-2}{n}[S-n(r-1)]\right\}^{2} \geqslant \frac{(n-2)^{2}}{n^{2}}\{n(n-1)(r-1)+S\}\{S-n(r-1)\} .
$$

Since $r \geqslant(n-2) /(n-1)$, then we get $r-1 \geqslant-1 /(n-1)$ and $n(r-1)+2$ $\geqslant(n-2) /(n-1)$, hence

$$
\begin{aligned}
n+n(r-1)-\frac{n-2}{n}[S-n(r-1)] \\
\quad \geqslant n+2(n-1)(r-1)-\frac{n-2}{n}\left[(n-1) \frac{n(r-1)+2}{n-2}+\frac{n-2}{n(r-1)+2}\right] \\
\quad=n+2(n-1)(r-1)-\frac{n-1}{n}[n(r-1)+2]-\frac{(n-2)^{2}}{n} \frac{1}{n(r-1)+2} \\
\quad=\frac{n^{2}-2(n-1)}{n}+(n-1)(r-1)-\frac{(n-2)^{2}}{n} \frac{1}{n(r-1)+2} \\
\geqslant \\
\geqslant \frac{n^{2}-2(n-1)}{n}-1-\frac{(n-2)^{2}}{n} \frac{n-1}{n-2}=0 .
\end{aligned}
$$

Obviously, by $(2.9)$ and $f^{2}=(n-1) / n[S-n(r-1)]$, we have $n(n-1)(r-1)$ $+S \geqslant 0, S-n(r-1) \geqslant 0$. Hence from (3.11) we have

$$
n+n(r-1)-\frac{n-2}{n}[S-n(r-1)] \geqslant \frac{n-2}{n} \sqrt{[n(n-1)(r-1)+S][S-n(r-1)]}
$$

that is

$$
P_{r}(S) \geqslant 0
$$

From (3.10),(3.13) we have $\operatorname{Ric}(v, v) \geqslant 0$ at all points of $M$.

CASE (i). When $S<(n-1)(n(r-1)+2) /(n-2)+(n-2) /(n(r-1)+2)$ holds at all points of $M$, or it holds at somewhere of $M$, then we all have the fundamental group of $M$ is finite. 
In fact, when $S<(n-1)(n(r-1)+2) /(n-2)+(n-2) /(n(r-1)+2)$ holds at all points of $M$, from the assertions above, we have $\operatorname{Ric}(v, v)>0$ at all points of $M$. Hence by the classical Myers Theorem, we know that the fundamental group of $M$ is finite.

When $S<(n-1)(n(r-1)+2) /(n-2)+(n-2) /(n(r-1)+2)$ holds at some points of $M$, from the assertions above, we know that $\operatorname{Ric}(v, v)>0$ holds at such points of $M$. From Lemma 5, we know that there exists a metric on $M$ such that the Ricci curvature is positive on $M$. Hence, we also know that the fundamental group of $M$ is finite.

Therefore, the proof of Theorem in the case where $n=3$ following directly from the Hamilton Theorem (see [6]) which states that a compact and connected oriented Riemannian 3-manifold with positive Ricci curvature is diffeomorphic to a spherical space form.

Now, we consider the case $n \geqslant 4$. Taking any positive integers $p, q$ such that $p+q$ $=n, 1<p, q<n-1$. Then $p q=p(n-p)=n+(p-1) n-p^{2} \geqslant n+(p-1)(p+2)-p^{2}$ $=n+(p-2) \geqslant n$. Let $T=\operatorname{tr}\left(h_{i j}\right)=\sum_{s=1}^{p} h_{s s}+\sum_{t=p+1}^{n} h_{t t}, \widetilde{S}=\sum_{i}\left(h_{i i}\right)^{2}, S=\sum_{i, j}\left(h_{i j}\right)^{2}$, then we have

$$
2 \sum_{s=1}^{p} \sum_{t=p+1}^{n}\left(h_{s t}\right)^{2}+\frac{p q}{n} \widetilde{S} \leqslant \frac{p q}{n}\left[2 \sum_{s=1}^{p} \sum_{t=p+1}^{n}\left(h_{s t}\right)^{2}+\widetilde{S}\right] \leqslant \frac{p q}{n} S .
$$

When $p \geqslant q,|p-q|=p-q=n-2 q<n-2$, when $p<q,|p-q|=q-p=n-2 p$ $<n-2$, therefore, $|p-q|<n-2$ for all $p, q$ and $\sqrt{p q} \geqslant \sqrt{n}>\sqrt{n-1}$.

By Lemma 2,(3.14) and $\widetilde{S} \leqslant S$, we make use of the same calculation for general submanifold in [12], we get for hypersurface that

$$
\begin{aligned}
\sum_{s=1}^{p} \sum_{t=p+1}^{n} & \left(2\left|B\left(e_{s}, e_{t}\right)\right|^{2}-\left\langle B\left(e_{s}, e_{s}\right), B\left(e_{t}, e_{t}\right)\right\rangle\right) \\
& =2 \sum_{s=1}^{p} \sum_{t=p+1}^{n}\left(h_{s t}\right)^{2}-\sum_{s=1}^{p} \sum_{t=p+1}^{n} h_{s s} h_{t t} \\
& =2 \sum_{s=1}^{p} \sum_{t=p+1}^{n}\left(h_{s t}\right)^{2}+\left(\sum_{s=1}^{p} h_{s s}\right)^{2}-T\left(\sum_{s=1}^{p} h_{s s}\right) \\
& \leqslant 2 \sum_{s=1}^{p} \sum_{t=p+1}^{n}\left(h_{s t}\right)^{2}+\frac{p q}{n} \widetilde{S}-\frac{2 p q}{n^{2}} T^{2}+\frac{|p-q|}{n^{2}} \sqrt{p q}|T| \sqrt{n \widetilde{S}-T^{2}} \\
& \leqslant \frac{p q}{n} S-\frac{2 p q}{n^{2}} T^{2}+\frac{|p-q|}{n^{2}} \sqrt{p q}|T| \sqrt{n \widetilde{S}-T^{2}} \\
& \leqslant \frac{p q}{n}\left[S-2 n H^{2}+\frac{|p-q|}{\sqrt{p q}}|H| \sqrt{n S-n^{2} H^{2}}\right] \\
& <\frac{p q}{n}\left[S-2 n H^{2}+\frac{\sqrt{n}(n-2)}{\sqrt{n-1}}|H| \sqrt{S-n^{2} H^{2}}\right]
\end{aligned}
$$




$$
=-\frac{p q}{n}\left[n+n H^{2}-\frac{n(n-2)}{\sqrt{n(n-1)}}|H| f-f^{2}\right]+p q .
$$

Therefore, from (3.6) or (3.7) and (3.13) we have

$$
\sum_{s=1}^{p} \sum_{t=p+1}^{n}\left(2\left|B\left(e_{s}, e_{t}\right)\right|^{2}-\left\langle B\left(e_{s}, e_{s}\right), B\left(e_{t}, e_{t}\right)\right\rangle\right)<-\frac{p q}{n} P_{r}(S)+p q<p q .
$$

Hence from Lemma $3 H_{p}(M, Z)=H_{q}(M, Z)=0$, for all $1<p, q<n-1, p+q=n$. Since $H_{n-2}(M, Z)=0$, taking the same discussion in [10], by the universal coefficient theorem $H^{n-1}(M, Z)$ has no torsion and consequently $H_{1}(M, Z)$ has no torsion by Poincare duality. By our assumption, since the fundamental group $\pi_{1}(M)$ of $M$ is finite, hence $H_{1}(M, Z)=0$, so $M$ is a homology sphere. The above arguments can be applied to the universal covering $\widetilde{M}$ of $M$. Since $\widetilde{M}$ is a homology sphere which is simple connected, that is $\pi_{1}(\widetilde{M})=0$, it is also a homotopy sphere. By the generalised Poincare conjecture (Smale $n \geqslant 5$, Freedman $n=4$ ) we have $\widetilde{M}$ is homeomorphic to a sphere and hence we have a homotopy sphere $M$ which is covered by a sphere $\widetilde{M}$, so by a result of Sjerve [13] we have $\pi_{1}(M)=0$, and hence $M$ is homeomorphic to a sphere.

CASE (ii). $S \equiv(n-1)(n(r-1)+2) /(n-2)+(n-2) /(n(r-1)+2)$ on $M$, from the discussion above this is equivalent to $P_{r}(S)=0$. Since the scalar curvature $n(n-1) r$ is constant, thus $S$ is constant, and by (2.9) $H$ is also constant. Hence the equalities in (3.8),(3.5) and (2.21) in Lemma 4 hold. If $r \geqslant(n-2) /(n-1)$, since $S=(n-1)(n(r-1)+2) /(n-2)+(n-2) /(n(r-1)+2)>(n-1)(n(r-1)+2) /(n-2)$ $>n(r-1)$, then $f^{2}=(n-1) / n[S-n(r-1)] \neq 0$, that is $M$ is not umbilical. When the equality in (2.21) holds, by Lemma $4 M$ is of only two distinct principal curvatures, one with multiplicity 1 and the other with multiplicity $n-1$. After renumberation if necessary, we can assume that $\lambda=\lambda_{1}=\cdots=\lambda_{n-1}, \mu=\lambda_{n}$. When the equalities in (3.8) or(3.5) hold. We have

$$
h_{i j k}=0 \text {. }
$$

Choose a local frame of orthonormal vector fields such that $h_{i j}=\lambda_{i} \delta_{i j}$, from (2.6) $\omega_{i i}=0$. Let $i=j$ in (2.12), from (3.16) and (2.12) we have $0=d \lambda_{i}-2 \sum_{k} h_{i k} \omega_{k i}=d \lambda_{i}$, hence $\lambda_{i}$ is constant, again from (2.12) we have

$$
0=\lambda_{i} \omega_{i j}+\lambda_{j} \omega_{j i}=\left(\lambda_{i}-\lambda_{j}\right) \omega_{i j}
$$

then for $\lambda_{i} \neq \lambda_{j}$

$$
\omega_{i j}=0
$$

From (2.7) and (3.18), if $\lambda_{i} \neq \lambda_{j}$, then

$$
0=d \omega_{i j}=-\sum_{k} \omega_{i k} \wedge \omega_{k j}+\frac{1}{2} \sum_{k, l} R_{i j k l} \omega_{k} \wedge \omega_{l}
$$


If for some $k$ such that $\omega_{i k} \neq 0$ and $\omega_{k j} \neq 0$,then by (3.17) we have $\lambda_{i}=\lambda_{k}=\lambda_{j}$, this contradicts to $\lambda_{i} \neq \lambda_{j}$, so $\sum_{k, l} R_{i j k l} \omega_{k} \wedge \omega_{l}=0$, thus, if $\lambda_{i} \neq \lambda_{j}$ we have

$$
R_{i j k l}=0 .
$$

From(3.20) and the Guass equation (2.8) we have $1+\lambda_{i} \lambda_{j}=0$ for $\lambda_{i} \neq \lambda_{j}$, that is

$$
1+\lambda \mu=0
$$

From (2.9) we have

$$
\mu=\frac{n(r-1)}{2 \lambda}-\frac{n-2}{2} \lambda .
$$

Hence from (3.21),(3.22) we get $\lambda^{2}=(n(r-1)+2) /(n-2)$ and $\mu^{2}=(n-2) /$ $(n(r-1)+2)$. Thus we get that $M$ is isoparametric. Therefore, $M$ is isometric to the Riemannian prodnct $S^{1}\left(\sqrt{1-c^{2}}\right) \times S^{n-1}(c)$, where $c^{2}=(n-2) /(n r)$. The Theorem is proved.

\section{REFERENCES}

[1] H. Alencar and M.P. do Carmo, 'Hypersurfaces with constant mean curvature in spheres', Proc. Amer. Math. Soc. 120 (1994), 1223-1229.

[2] T. Aubin, Some nonlinear problems in Riemannian geometry (Springer-Verlag, Berlin, New York, 1998).

[3] S.Y. Cheng and S.T. Yau, 'Hypersurfaces with constant scalar curvature', Math. Ann. 225 (1977), 195-204.

[4] Q.M. Cheng, 'Hypersurfaces in a unit sphere $S^{n+1}(1)$ with constant scalar curvature', $J$. London Math. Society 64 (2001), 755-768.

[5] K.R. Cai, 'Topology of certain closed submanifolds in a Euclidean space', Chinese Ann. Math. Ser. A 8 (1987), 234-241.

[6] R. Hamilton, 'Three manifolds with positive Ricci curvature', J. Differential Geom. 17 (1982), 255-306.

[7] H. Li, 'Hypersurfaces with constant scalar curvature in space forms', Math. Ann. 305 (1996), 665-672.

[8] H. Lawson and J. Simons, 'On stable currents and their application to global problems in real and complex geometry', Ann. of Math. 98 (1973), 427-450.

[9] P.F. Leung, 'An estimate on the Ricci curvature of a submanifold and some applications', Proc. Amer. Math. Soc. 114 (1992), 1051-1061.

[10] P.F. Leung, 'Minimal submanifolds in sphere', Math. Z. 183 (1983), 75-86.

[11] M.Okumura, 'Hypersurfaces and a pinching problem on the second fundamental tensor', Amer. J. Math. 96 (1974), 207-213.

[12] K. Shiohama and $\mathrm{H}$. Xu, 'The topological sphere theorem for complete submanifolds', Compositio Math. 107 (1997), 221-232. 
[13] D. Sjerve, 'Homology spheres which are covered by spheres', J. London Math. Soc. (2) 6 (1973), 333-336.

Department of Applied Mathematics

Xidian University

Xi'an 710071

Shaanxi

Peoples Republic of China

e-mail: xysxssc@yahoo.com.cn
Department of Mathematics

Xianyang Teachers' University

Xianyang 712000

Shaanxi

Peoples Republic of China 